Ana I. Pereira - Florbela P. Fernandes .

João P. Coelho · João P. Teixeira .

Maria F. Pacheco · Paulo Alves ·

Rui P. Lopes (Eds.)

Optimization, Learning

Algorithms and Applications

First International Conference, OL2A 2021

Bragança, Portugal, July 19-21, 2021

Revised Selected Papers

望 Springer 


\section{Editors}

Ana I. Pereira

Instituto Politécnico de Bragança

Bragança, Portugal

João P. Coelho

Instituto Politécnico de Bragança

Bragança, Portugal

Maria F. Pacheco

Instituto Politécnico de Bragança

Bragança, Portugal

Rui P. Lopes (D)

Instituto Politécnico de Bragança

Bragança, Portugal
Florbela P. Fernandes (D)

Instituto Politécnico de Bragança

Bragança, Portugal

João P. Teixeira (1D)

Instituto Politécnico de Bragança

Bragança, Portugal

Paulo Alves

Instituto Politécnico de Bragança

Bragança, Portugal

ISSN 1865-0929

ISSN 1865-0937 (electronic)

Communications in Computer and Information Science

ISBN 978-3-030-91884-2

ISBN 978-3-030-91885-9 (eBook)

https://doi.org/10.1007/978-3-030-91885-9

\section{(C) Springer Nature Switzerland AG 2021}

This work is subject to copyright. All rights are reserved by the Publisher, whether the whole or part of the material is concerned, specifically the rights of translation, reprinting, reuse of illustrations, recitation, broadcasting, reproduction on microfilms or in any other physical way, and transmission or information storage and retrieval, electronic adaptation, computer software, or by similar or dissimilar methodology now known or hereafter developed.

The use of general descriptive names, registered names, trademarks, service marks, etc. in this publication does not imply, even in the absence of a specific statement, that such names are exempt from the relevant protective laws and regulations and therefore free for general use.

The publisher, the authors and the editors are safe to assume that the advice and information in this book are believed to be true and accurate at the date of publication. Neither the publisher nor the authors or the editors give a warranty, expressed or implied, with respect to the material contained herein or for any errors or omissions that may have been made. The publisher remains neutral with regard to jurisdictional claims in published maps and institutional affiliations.

This Springer imprint is published by the registered company Springer Nature Switzerland AG The registered company address is: Gewerbestrasse 11, 6330 Cham, Switzerland 


\section{Preface}

The volume CCIS 1488 contains the refereed proceedings of the International Conference on Optimization, Learning Algorithms and Applications (OL2A 2021), an event that, due to the COVID-19 pandemic, was held online.

OL2A 2021 provided a space for the research community on optimization and learning to get together and share the latest developments, trends, and techniques as well as develop new paths and collaborations. OL2A 2021 had more than 400 participants in an online environment throughout the three days of the conference (July 19-21, 2021), discussing topics associated to areas such as optimization and learning and state-of-the-art applications related to multi-objective optimization, optimization for machine learning, robotics, health informatics, data analysis, optimization and learning under uncertainty, and the Fourth Industrial Revolution.

Four special sessions were organized under the following topics: Trends in Engineering Education, Optimization in Control Systems Design, Data Visualization and Virtual Reality, and Measurements with the Internet of Things. The event had 52 accepted papers, among which 39 were full papers. All papers were carefully reviewed and selected from 134 submissions. All the reviews were carefully carried out by a Scientific Committee of $61 \mathrm{PhD}$ researchers from 18 countries.

July 2021

Ana I. Pereira 


\section{Organization}

\section{General Chair}

Ana Isabel Pereira

Polytechnic Institute of Bragança, Portugal

\section{Organizing Committee Chairs}

Florbela P. Fernandes

João Paulo Coelho

João Paulo Teixeira

M. Fátima Pacheco

Paulo Alves

Rui Pedro Lopes

\section{Scientific Committee}

Ana Maria A. C. Rocha

Ana Paula Teixeira

André Pinz Borges

Andrej Košir

Arnaldo Cândido Júnior

Bruno Bispo

Carmen Galé

B. Rajesh Kanna

C. Sweetlin Hemalatha

Damir Vrančić

Daiva Petkeviciute

Diamantino Silva Freitas

Esteban Clua

Eric Rogers

Felipe Nascimento Martins

Gaukhar Muratova

Gediminas Daukšys

Glaucia Maria Bressan

Humberto Rocha

José Boaventura-Cunha

José Lima

Joseane Pontes

Juani Lopéz Redondo
Polytechnic Institute of Bragança, Portugal

Polytechnic Institute of Bragança, Portugal

Polytechnic Institute of Bragança, Portugal

Polytechnic Institute of Bragança, Portugal

Polytechnic Institute of Bragança, Portugal

Polytechnic Institute of Bragança, Portugal 


Jorge Ribeiro
José Ramos
Kristina Sutiene
Lidia Sánchez
Lino Costa
Luís Coelho
Luca Spalazzi
Manuel Castejón Limas
Marc Jungers
Maria do Rosário de Pinho
Marco Aurélio Wehrmeister
Mikulas Huba

Michał Podpora

Miguel Ángel Prada

Nicolae Cleju

Paulo Lopes dos Santos

Paulo Moura Oliveira

Pavel Pakshin

Pedro Luiz de Paula Filho

Pedro Miguel Rodrigues

Pedro Morais

Pedro Pinto

Rudolf Rabenstein

Sani Rutz da Silva

Sara Paiva

Sofia Rodrigues

Sławomir Stępień

Teresa Paula Perdicoulis

Toma Roncevic

Vitor Duarte dos Santos

Wojciech Paszke

Wojciech Giernacki
Polytechnic Institute of Viana do Castelo, Portugal

NOVA University Lisbon, Portugal

Kaunas University of Technology, Lithuania

University of León, Spain

University of Minho, Portugal

Polytecnhic Institute of Porto, Portugal

Marche Polytechnic University, Italy

University of León, Spain

Université de Lorraine, France

University of Porto, Portugal

Federal University of Technology - Paraná, Brazil

Slovak University of Technology in Bratislava, Slovakia

Opole University of Technology, Poland

University of León, Spain

Technical University of Iasi, Romania

University of Porto, Portugal

University of Trás-os-Montes and Alto Douro, Portugal

Nizhny Novgorod State Technical University, Russia

Federal University of Technology - Paraná, Brazil

Catholic University of Portugal, Portugal

Polytechnic Institute of Cávado e Ave, Portugal

Polytechnic Institute of Viana do Castelo, Portugal

Friedrich-Alexander-University of Erlangen-Nürnberg, Germany

Federal University of Technology - Paraná, Brazil

Polytechnic Institute of Viana do Castelo, Portugal

Polytechnic Institute of Viana do Castelo, Portugal

Poznan University of Technology, Poland

University of Trás-os-Montes and Alto Douro, Portugal

University of Split, Croatia

NOVA University Lisbon, Portugal

University of Zielona Gora, Poland

Poznan University of Technology, Poland 
Optimizing Data Transmission in a Wireless Sensor Network Based on LoRaWAN Protocol

Thadeu Brito, Matheus Zorawski, João Mendes,

Beatriz Flamia Azevedo, Ana I. Pereira, José Lima, and Paulo Costa

Indoor Location Estimation Based on Diffused Beacon Network . . . . . . . . . 294 André Mendes and Miguel Diaz-Cacho

SMACovid-19 - Autonomous Monitoring System for Covid-19 . . . . . . . . 309 Rui Fernandes and José Barbosa

\section{Optimization in Control Systems Design}

Economic Burden of Personal Protective Strategies for Dengue Disease:

an Optimal Control Approach

Artur M. C. Brito da Cruz and Helena Sofia Rodrigues

ERP Business Speed - A Measuring Framework ................. 336 Zornitsa Yordanova

BELBIC Based Step-Down Controller Design Using PSO

João Paulo Coelho, Manuel Braz-César, and José Gonçalves

Robotic Welding Optimization Using A* Parallel Path Planning Tiago Couto, Pedro Costa, Pedro Malaca, Daniel Marques, and Pedro Tavares

\section{Deep Learning}

Leaf-Based Species Recognition Using Convolutional Neural Networks Willian Oliveira Pires, Ricardo Corso Fernandes Jr., Pedro Luiz de Paula Filho, Arnaldo Candido Junior, and João Paulo Teixeira

Deep Learning Recognition of a Large Number of Pollen Grain Types . . . . . 381 Fernando C. Monteiro, Cristina M. Pinto, and José Rufino

Predicting Canine Hip Dysplasia in X-Ray Images Using Deep Learning . . . . . . Daniel Adorno Gomes, Maria Sofia Alves-Pimenta, Mário Ginja, and Vitor Filipe

Convergence of the Reinforcement Learning Mechanism Applied to the Channel Detection Sequence Problem 


\title{
BELBIC Based Step-Down Controller Design Using PSO
}

\author{
João Paulo Coelho ${ }^{1,2(\bowtie)}$, Manuel Braz-César ${ }^{1,3}$, and José Gonçalves ${ }^{1,2}$ \\ 1 Instituto Politécnico de Bragança, Escola Superior de Tecnologia e Gestão, \\ Campus de Sta. Apolónia, 5300-253 Bragança, Portugal \\ \{jpcoelho, brazcesar, goncalves\}@ipb.pt \\ 2 CeDRI - Centro de Investigação em Digitalização e Robótica Inteligente, \\ Campus de Sta. Apolónia, 5300-253 Bragança, Portugal \\ 3 CONSTRUCT Institute of R\&D in Structures and Construction, \\ Campus da FEUP, 4200-465 Porto, Portugal
}

\begin{abstract}
This article presents a comparison between a common type III controller and one based on a brain emotional learning paradigm (BELBIC) parameterized using a particle swarm optimization algorithm (PSO). Both strategies were evaluated regarding the set-point accuracy, disturbances rejection ability and control effort of a DC-DC buck converter. The simulation results suggests that, when compared to the common controller, the BELBIC leads to an increase in both set-point tracking and disturbances rejection ability while reducing the dynamics of the control signal.
\end{abstract}

Keywords: Optimisation $\cdot \mathrm{BELBIC} \cdot$ Buck converter $\cdot$ PSO

\section{Introduction}

Conversion between different voltages values is amongst the most common operations found in electronics. For example, many battery-operated devices such as laptops and mobile devices, are capable of switching between different voltage values in order to optimize the use of the battery. The $5 \mathrm{~V}$ constant core voltage found of 1970's microprocessors has evolved for today's processors to scalable core supply voltage that can reach values lower than one volt. This voltage scaling task can be performed dynamically at the software or firmware levels by both the operating system or BIOS. Moreover, a point-to-load approach used in the motherboard of modern microprocessor devices has led to the inclusion of a large number of small power supplies scattered along the main board. Reducing the power dissipated in the form of heat is an important goal which lead to an increase in efficiency, small form factors by discarding the use of large heat sinks and an extension of battery life which is a key factor for all mobile devices. This can be attained by resorting to a class of circuits known by switch-mode power supplies where the voltage conversion takes place by periodically switching transistors, embedded in RLC networks, between their on and off states. The

(C) Springer Nature Switzerland AG 2021

A. I. Pereira et al. (Eds.): OL2A 2021, CCIS 1488, pp. 345-356, 2021.

https://doi.org/10.1007/978-3-030-91885-9_25 
input-to-output voltage ratio depends on the duty cycle imposed to those switching devices by a controller. This controller operates in closed-loop by sampling the output voltage and comparing it with the desired output voltage value and the difference between those two values will be used to establish the switching duty-cycle. The block diagram presented in Fig. 1 illustrate this methodology.

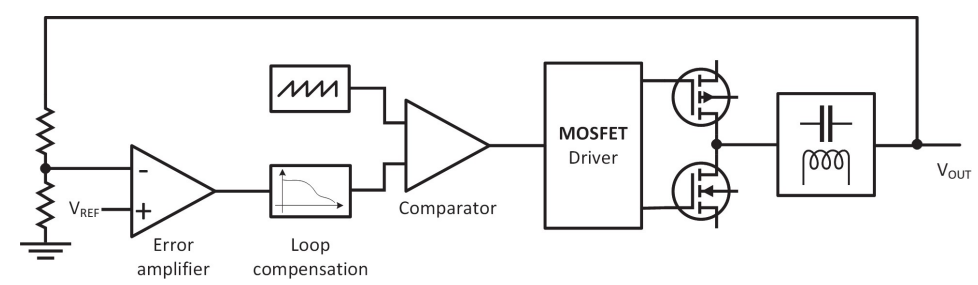

Fig. 1. Typical feedback control architecture used in DC/DC converters.

Often, in practice, a current loop is also added in order to enable currentmode control. This additional control layer enables overcurrent protection and reduces the sensitivity of the voltage controller to the capacitor's ESR. However, in this paper, only the voltage-mode control is taken into consideration. Voltagemode control resort to feedback to keep constant the output voltage despite unwanted disturbances. The loop compensation network associated to the error amplifier can be of type I, II or III. Type I is a simple pole at the origin and type II expand it by including a zero and a high-frequency pole which can leading to a phase increase of $90^{\circ}$. Finally, type III adds two poles and two zeros to the pole at zero which promotes an increase in phase margin.

Those loop compensation circuits are tuned to perform well in a given nominal system operating point. However, if the system deviates from this point, the controller performance can become very poor. For example, when the power supply shift between continuous to discontinuous conduction mode. Hence, adaptiveness and learning ability must be included in the controller in order for it to be able to perform well in a large dynamic range and under the presence of system changes.

This work proposes an alternative controller structure applied to regulate the operation of a DC to DC buck converter. In particular, it will rely on the use of control paradigm inspired on the brain emotional learning ability (BELBIC) to promote adaption to operating point changes. Conceptually, this controller is inspired by the brain's limbic system and, when compared to the typical buck converter controller, its most notorious property is the ability to keep learning while in operation. The use of BELBIC was already applied within the power electronics context. In [1], a brain emotional learning approach was employed in the context of a maximum power-point tracking algorithm applied to solar energy conversion. Additionally, in [2], a BELBIC controller was applied to control a 
buck DC-DC converter. However, the controller parameter were obtained empirically and no comparison with other techniques was carried out. In this paper, the buck converter is also addressed but the BELBIC parameters were computed using the particle swarm optimization algorithm (PSO). Moreover, comparison of closed loop response with typical loop compensators was performed.

This document is divided into four sections. After this first introductory section, the mathematical formulation of a step down converter is described in Sect. 2. Then, the generic BELBIC structure is presented in Sect. 3 and a general overview of the PSO algorithm is presented in Sect. 4. Details and results regarding the controller implementation is the aim of Sect. 5 where a performance comparison was carried out having a Type II controller as benchmark. Finally, Sect. 6 presents both the conclusions and final remarks.

\section{The Step-Down (Buck) Converter}

The DC-DC buck converter used in this work assumes the asynchronous architecture depicted in Fig. 2. The two switching elements are a MOSFET and a diode. The MOSFET gate will be driven by a pulse-width modulation (PWM) circuit that, for simplicity, is not shown.

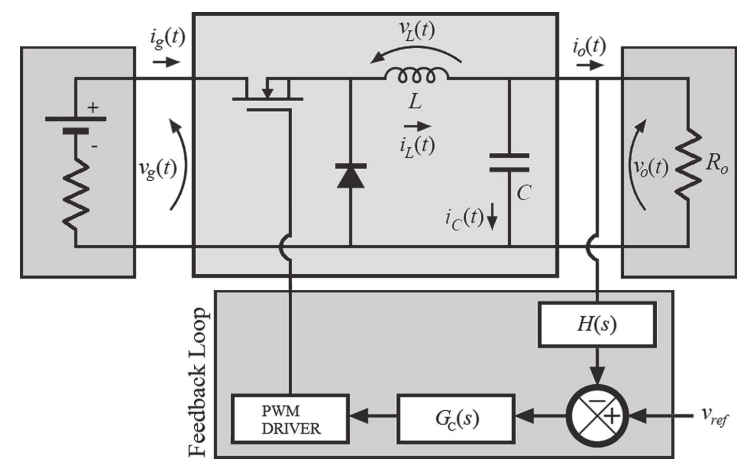

Fig. 2. General schematic of a DC-DC step-down converter built around a MOSFET and a diode as switching elements.

In this figure, $H(s)$ denotes the voltage sensor transfer function and $G_{C}(s)$ the controller transfer function. The MOSFET gate is driven by a pulse width modulation circuit that generates a square wave whose duty-cycle is proportional to a voltage signal applied to its input.

Considering both steady state and converter's continuous conduction operating mode, the average voltage across the inductor assumes the value zero. At the same time, the average current value across the capacitor, over one switching period, is also zero. 
Assuming small magnitudes of the disturbances, compared to the DC quiescent values, it is possible to obtain the following differential equations [3]:

$$
\begin{gathered}
L \frac{d}{d t} \hat{i}_{L}(t)=D \hat{v}_{g}(t)+\hat{d}(t) V_{g}-\hat{v}_{o}(t) \\
C \frac{d}{d t} \hat{v}_{o}(t)=\hat{i}_{L}(t)-\frac{\hat{v}_{o}(t)}{R_{o}} \\
\hat{i}_{g}=D \hat{i}_{L}(t)+i_{L}(t) \hat{d}(t)
\end{gathered}
$$

where the hat symbol over the variables denotes a small disturbance around the variable's operating point and $D$ is the PWM duty cycle whose value is within the interval $[0,1]$.

After applying the Laplace transform to the previous set of differential equations, the transfer function between the output and the command signal, denoted by $G_{v_{o} d}(s)$, is:

$$
G_{v_{o} d}(s)=\frac{\hat{v}_{o}(s)}{\hat{d}(s)}=\frac{R_{o} V_{g}}{L C R_{o} s^{2}+L s+R_{o}}
$$

and from input to output voltage, $G_{v_{o} v_{g}}(s)$, defined as:

$$
G_{v_{o} v_{g}}(s)=\frac{\hat{v}_{o}(s)}{\hat{v}_{g}(s)}=\frac{R_{o} D}{L C R_{o} s^{2}+L s+R_{o}}
$$

Without input voltage disturbances or load changes, the converter is able to operate in open-loop. However, the output value can fluctuates in the presence of load changes of other disturbances as input voltage drops/rises or shifts in the components nominal values due to several factors such as aging. Thus, a closed-loop controller must be added in order to regulate the switched converter voltage output.

Typically, type I, II or III loop compensator structures are chosen to carry out this task and can be designed using the previously defined transfer functions. However, it is worth to notice that those transfer functions are only approximations and assumes small disturbances around a given operating point. For this reason, the behaviour of the switching converter can be very different outside the defined zone. Specially, if due to small loads, the converter settles to work in discontinuous conduction mode. Fixed poles-zeros controller are unable to achieve good performance in the presence of severe changes in the system dynamic behaviour. Other approach is to enable the controller to learn and use this knowledge to self adjust its behaviour in order to increase the overall performance within a broader range of operating points. In this work, this feature will be attained by resorting to a soft-computing paradigm known by BELBIC and briefly described in the following section.

\section{The BELBIC Controller}

From an engineering point-of-view, analysis of the solutions produced by nature to overcome the animal species adaption problems, have led to an increasing 
tendency to introduce bio-morphism and bio-mimicry in many different computational tools [4]. For example, biological inspiration can be tracked in applications such as machine cooperation, speech recognition, text recognition and self-assembly nanotechnology just to name a few. All those examples have, in fact that all rely on algorithms which have the capability of adaptation and learning. Indeed, and in the biological realm, learning is one of the most important factors for the endurance of all species. Learning allow the organisms to adjust themselves to cope with changes in environmental and operating conditions. This robustness is a desired characteristic in engineering applications since the operating conditions of a product are never static. For this reason, simplified approaches of some natural learning processes have been adapted to serve in many engineering problems.

In the particular case of humans, learning takes place within generations and across generations at many different levels. We are not talking only about intellectual learning but also about the learning that is passed through our genome. All this information shapes the actions of an individual when subject to a set of environmental stimuli. At the mental level, reasoning is not the only driving action when decision-making is concerned. Human reactions strongly depend also on emotions and they play an important role in our everyday life and have been a valuable asset in our survival and adaptation.

Is generally considered true that emotions were included during the evolutionary stage as a way to reduce the human's reaction time. That is, rather than using the intellect to process information and generate actions, which would take time, the reaction by emotion would be much faster. Emotions can then be viewed as an automatic behaviour that seeks to improve survival by increasing the ability to react fast in the presence of threats. It seems that the overall set of possible emotions are predefined in our genome. However, they can then be utterly modified based on the person's individual experience.

Psychology and neural sciences circumscribe emotional activity to a set of distinct brain regions gathered in what is known as the limbic region. Besides emotions, the limbic system manages a distinct number of other functions such as behaviour, motivation and has an important role in memory formation tasks. At the present, it is still not consensual in the scientific community about which brain areas should be included in the limbic system. However, it is commonly accepted that the thalamus, hypothalamus, hippocampus and amygdala are the main brain structures in the limbic system. Details regarding the role played by each one of those cortical areas are outside the scope of this work. Instead, the objective is to convert the limbic system behaviour, from a high-level abstraction angle, and frame it in the context of computational intelligence. The first step toward this approach was carried out by [5] by presenting a first mathematical approach to describe the behaviour of the brain's emotional learning (BEL) process. The idea of applying this learning algorithm in the automatic control area was provided, a couple of years later, by [6]. The junction of BEL with control systems design has led to the concept known as "brain emotional learning-based 
intelligent control" (BELBIC). Further details regarding the operational details of this control method can be found at [7-10].

The major pitfalls of BELBIC regards the choice of both emotional and sensory signals in order to maximize the control system performance. Besides that, several tuning parameters, such as the learning rate of the amygdala and orbitofrontal processing units, must be found to achieve an acceptable controller behaviour. The values for such parameters are commonly found by trial-anderror which can be cumbersome and lead to suboptimal solutions. For those reasons, other tuning methods have been presented [11-13] among them the use of evolutionary based algorithms [14-18].

Due to its ability to provide good results for non-convex problems, evolutionary algorithms have been employed in a myriad of different applications. For this reason, in this work the BELBIC controller will be tuned by resorting to the particle swarm optimization algorithm. A short overview on this method is provided in the following section and further details on the methodology will be described in Sect. 5 .

\section{The PSO Algorithm}

The particle swarm optimisation (PSO) algorithm is fundamentally based on the social behaviour of animals that moves in herds or flocks [19]. In this algorithm, a set of particles, representing potential problem solutions moves through the search space according to a given position vector $\mathbf{x}_{i}(t)=$ $\left\{x_{i 1}(t), x_{i 2}(t), \cdots, x_{i n}(t)\right\}$ and velocity $\mathbf{v}_{i}(t)=\left\{v_{i 1}(t), v_{i 2}(t), \cdots, v_{i n}(t)\right\}$ where $t$ denotes the current evolutionary iteration and $n$ the number of particles.

The PSO dynamics is governed by individual and social knowledge. That is, a given particle movement is due to it's own experience and from social information sharing. In [19] this concept was mathematically expressed by the following set of equations:

$$
\begin{aligned}
& c o_{i d}(t)=\left(p_{i d}(t)-x_{i d}(t)\right) \\
& s o_{i d}(t)=\left(p_{g d}(t)-x_{i d}(t)\right)
\end{aligned}
$$

where $c o_{i d}(t)$ is the cognition-only value associated to the $\mathrm{d}^{\text {th }}$ dimension of the $i^{t h}$ particle and $s o_{i d}(t)$ is the social-only component of the same individual.

The momentum and position of a given particle are computed by:

$$
\begin{gathered}
v_{i d}(t+1)=v_{i d}(t)+\varphi_{1} \cdot c o_{i d}(t)+\varphi_{2} . s o_{i d}(t) \\
x_{i d}(t+1)=x_{i d}(t)+v_{i d}(t+1)
\end{gathered}
$$

where $p_{i d}(t)$ concerns the best previous position of particle $i$ in the current iteration $t$ and $p_{g d}(t)$ denotes the global best particle within a given neighbourhood. The coefficient $\varphi_{1}$ is the cognitive constant and $\varphi_{2}$ is the social coefficient. Generally they are assumed to be uniformly distributed random numbers between zero and two. 
To guarantee admissibility and stability, both the particle position and velocity are bounded to maximum values. For this reason, the search space is always circumscribed and the maximum step that each particle can undergo during one iteration is constrained. The values for the maximum or minimum particle position are problem dependent. Moreover, the maximum velocity should not be too high or too low in order to avoid oscillations and local minima [20].

\section{Step-Down Control with BELBIC}

This section present the procedure behind the design a BELBIC controller for a DC-DC buck converter capable of generating a $5 \mathrm{~V}$ output regulated voltage from a $12 \mathrm{~V}$ unregulated voltage input. The electrical components nominal values are $L=20 \mathrm{mH}, C=50 \mu \mathrm{F}$ and a $50 \mathrm{kHz}$ switching frequency was considered. To reach the above referred nominal output voltage the duty-cycle $D$ must be roughly equal to $42 \%$. For this buck converter, a type III controller was designed in order to have zero steady state error, around $60^{\circ}$ of phase margin, $10 \mathrm{~dB}$ of gain margin and the open-loop frequency response describes a slope of $-20 \mathrm{~dB} /$ decade at the crossover frequency. Also, an overshoot lower than $0.5 \mathrm{~V}$ and a rise time smaller or equal to $2 \mathrm{~ms}$. Using the Bode plot reshaping technique, all those figures-of-merit could be attained by means a regulator with the following transfer function:

$$
G_{c}(s)=\frac{0.0317 s^{2}+63.4 s+5 \times 10^{4}}{s\left(s^{2}+20 s+100\right)}
$$

Figure 3 present the circuit implemented using Simulink ${ }^{\circledR}$ 's Simscape ${ }^{\circledR}$ toolbox. Using this framework, the buck converter non-linear nature is fully represented since each electronic and electrical components is accurately modelled taking into consideration its non-ideal characteristics.

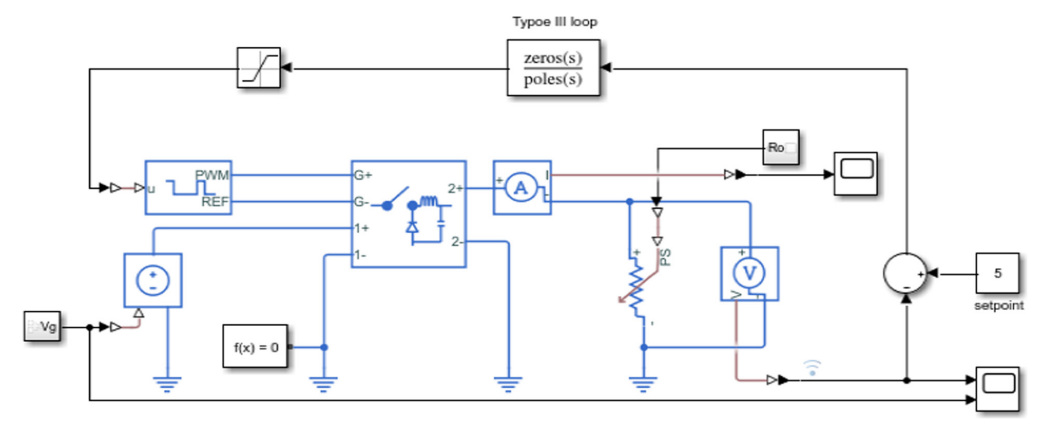

Fig. 3. Closed-loop implementation of the buck converter using SiMSCAPE ${ }^{\circledR}$ and a type III compensator. 
The simulation was carried out considering a $5 \mathrm{~V}$ sinusoidal disturbance, with $100 \mathrm{~Hz}$ frequency, superimposed over the $12 \mathrm{~V}$ supply voltage. Moreover, a $20 \%$ step load disturbance was applied at time instant $0.005 \mathrm{~s}$. The simulation was carried out within a time frame of $15 \mathrm{~ms}$ and the observed results are shown in Fig. 4.

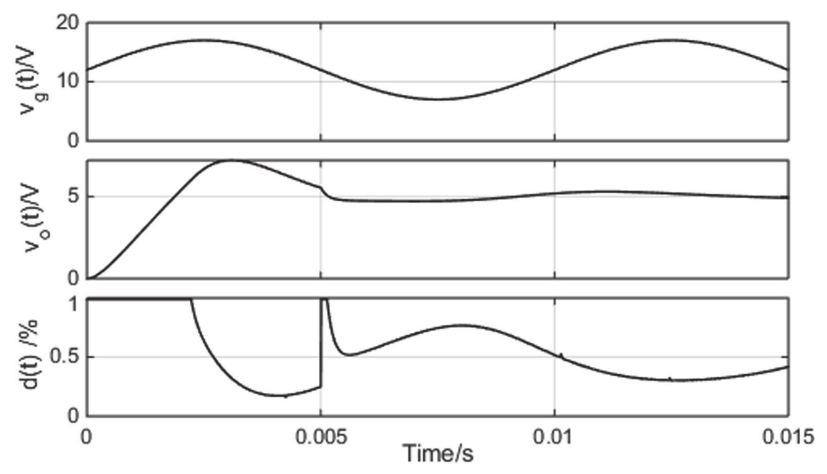

Fig. 4. Buck converter performance using a PID type controller: top - input voltage, middle - output voltage, bottom - control signal.

From the simulation result, it is possible to observe a performance degradation in both overshoot and bandwidth. Moreover, the set-point accuracy was compromised as can be seen by the low frequency signal overlapped into the output voltage. This closed-loop mismatch is due to several reasons: components losses, non-linearities, and model mismatches. For this reason, it is possible to conclude that this controller is unable to perform well in a broad range of changes in the system dynamics. Adaption is required which, in this work, is attained by means of using the BELBIC control strategy.

One of the major handicaps when dealing with a BELBIC controller concerns the appropriate definition of both emotional and sensory signals. In this work, the BELBIC SimULINK ${ }^{\circledR}$ toolbox [10] was utilized with the structure depicted in Fig. 5 where the stimulus signal was defined as:

$$
s(t)=w_{1} \cdot e(t)+w_{2} \cdot \int e(t) d t
$$

where $e(t)$ denotes the voltage tracking error.

The reward signal is defined by:

$$
r(t)=w_{3} \cdot e(t)+w_{4} \cdot u(t)
$$

where $u(t)$ concerns the control signal and $w_{i}$, for $i=1, . ., 4$, are weight factors that can be used to define the relative importance of each component.

Besides the weights $w_{i}$, for $i=1, \cdots, 4$ presented in (7) and (8), the BELBIC design process require also the definition of a set of parameters for it to operate 


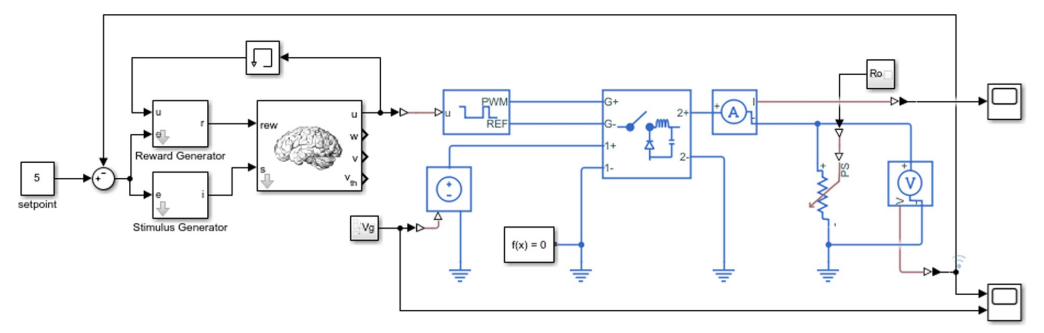

Fig. 5. Closed-loop implementation of the buck converter using SimSCAPE ${ }^{\circledR}$ and a BELBIC controller.

adequately. In particular the value of the amygdala and orbitofrontal learning rates $\alpha$ and $\beta$ respectively. Managing such a number of parameters using a trialand-error approach will be cumbersome at best. For this reason, in this work a PSO algorithm will be in charge of deriving the best controller parameters according to a given performance index.

In the current context, the performance is calculated by:

$$
f(\boldsymbol{\theta})=\phi(\boldsymbol{\theta}) \cdot \int_{0}^{t_{S}} e^{2}(\boldsymbol{\theta}, \tau) d \tau
$$

where $\boldsymbol{\theta}=\left[\alpha, \beta, w_{1}, w_{2}, w_{3}, w_{4}\right]$ denotes the controller parameters and $t_{S}$ the simulation time. The function $\phi(\boldsymbol{\theta})$ is used to penalizes solutions that result in control signals with amplitudes outside the actuator range. In the present case, $\phi(\boldsymbol{\theta})$ is defined as:

$$
\phi(\boldsymbol{\theta})=\left\{\begin{array}{l}
1, \quad \min (u(t)) \geq 0 \wedge \max (u(t)) \leq 1 \\
e^{\left.\left(\min (u(t))^{2}+\max (u(t))^{2}\right)\right)}+1, \text { otherwise }
\end{array}\right.
$$

for $t \in\left[0, t_{S}\right]$.

The PSO algorithm was run several times to search for a suitable solution $\boldsymbol{\theta}$ that minimizes the objective function $f(\boldsymbol{\theta})$. During the simulation, the system was exited using a random input voltage signal with values between $4 \mathrm{~V}$ and $15 \mathrm{~V}$ changing with a periodicity of $5 \mathrm{~ms}$. A swarm size of 30 particles was used and the simulation time was set to $100 \mathrm{~ms}$.

The best solution found, in this case $\alpha=0.0241, \beta=0.00985, w_{1}=6.54$, $w_{2}=21.3, w_{3}=0.0016$ and $w_{4}=0.21$, was used to parameterize the BEL controller which was then subjected to the same simulation conditions as the type III controller. The result can be seen from Fig. 6 .

As can be seen after analysing the plots of Fig. 6, the BELBIC controller was able to achieve a smaller settling time and lower overshoot. Moreover the steadystate error and disturbance rejection ability were also enhanced when compared to the PID controller. However, this improved response comes at the expense of a more complex and demanding tuning process. 


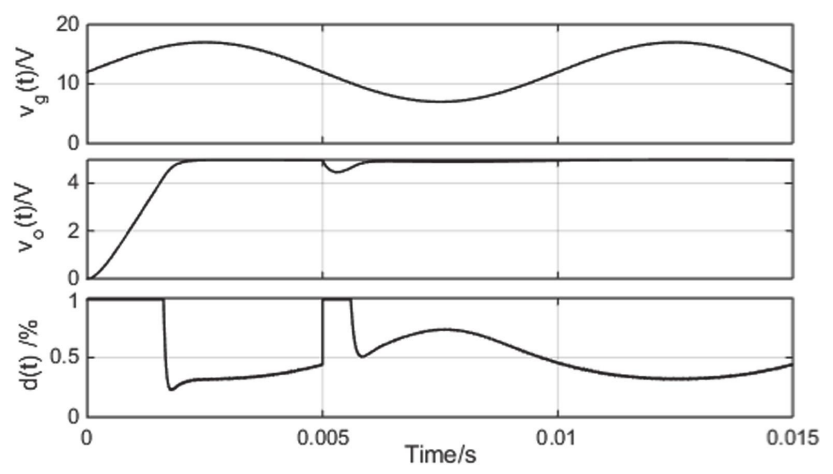

Fig. 6. Buck converter performance using a BELBIC type controller: top - input voltage, middle - output voltage, bottom - control signal.

A quantitative comparison between the PID and the BELBIC controllers, in the context of the addressed problem, can be made after computing the following two figures-of-merit:

$$
\begin{gathered}
\epsilon_{R M S}=\sqrt{\frac{1}{T} \int_{0}^{T} \varepsilon(t)^{2} d t} \\
\mu_{R M S}=\sqrt{\frac{1}{T} \int_{0}^{T}\left(\frac{d u(t)}{d t}\right)^{2} d t}
\end{gathered}
$$

The first is the root-mean-square value of the error signal $\varepsilon(t)$ taken along the simulation interval $[0, T]$ and the second, also the root-mean-square, but now of the control effort.

Regarding the PID controller, $\epsilon_{R M S}=1.35$ and $\mu_{R M S}=8.19 \times 10^{-3}$. For the BELBIC those values come down to $\epsilon_{R M S}=1.131$ and $\mu_{R M S}=7.12 \times 10^{-3}$. Those values reflect a $16 \%$ decrease in $\epsilon_{R M S}$ and an improvement of $13 \%$ in the control signal variability.

\section{Conclusion}

This paper has compared the performance between an ordinary type III controller, and a BELBIC controller applied to a DC-DC buck converter. Both strategies were evaluated regarding its abilities to maintain a stable voltage output in the presence of both input and load disturbances.

The obtained results suggest that, when compared to the classical controller, the BELBIC controller proves to be superior when considering both set-point tracking accuracy and disturbance rejection ability. Furthermore, these results are attained by means of lower control effort. 
Future work will consider the controller behaviour if the buck converter enters discontinuous conduction mode. A physical implementation of this solution is also an ongoing project and the controller performance will be compared with the one achieved by commercial devices such as the UC3845A chip.

\section{References}

1. Sankarganesh, R., Thangavel, S.: Performance analysis of various DC-DC converters with optimum controllers for PV applications. Res. J. Appl. Sci. Eng. Technol. 8, 929-941 (2014)

2. Khorashadizadeh, S., Mahdian, M.: Voltage tracking control of DC-DC boost converter using brain emotional learning. In: 4th International Conference on Control, Instrumentation, and Automation (ICCIA), pp. 268-272 (2016)

3. Erickson, R.W., Maksimovic, D.: Fundamentals of Power Electronics, 2nd edn. Springer, Boston (2001). https://doi.org/10.1007/b100747

4. Sarpeshkar, R.: Neuromorphic and Biomorphic Engineering Systems. McGraw-Hill Yearbook of Science and Technology. McGraw-Hill, New York (2009)

5. Balkenius, C., Morén, J.: A computational model of emotional learning in the amygdala. Cybern. Syst. 32(6), 611-636 (2001)

6. Lucas, C., Shahmirzadi, D., Sheikholeslami, N.: Introducing BELBIC: brain emotional learning based intelligent controller. Intell. Autom. Soft Comput. 10, 11-22 (2004)

7. Rouhani, H., Jalili, M., Araabi, B.N., Eppler, W., Lucas, C.: Brain emotional learning based intelligent controller applied to neurofuzzy model of micro-heat exchanger. Expert Syst. Appl. 32(3), 911-918 (2007)

8. Rahman, M.A., Milasi, R.M., Lucas, C., Araabi, B.N., Radwan, T.S.: Implementation of emotional controller for interior permanent-magnet synchronous motor drive. IEEE Trans. Ind. Appl. 44(5), 1466-1476 (2008)

9. Nahian, S.A., Truong, D.Q., Ahn, K.K.: A self-tuning brain emotional learning based intelligent controller for trajectory tracking of electrohydraulic actuator. J. Syst. Control Eng. 228, 461-475 (2014)

10. Coelho, J.P., Pinho, T.M., Boaventura-Cunha, J., de Oliveira, J.B.: A new brain emotional learning Simulink $(\mathbb{B}$ toolbox for control systems design. IFACPapersOnLine 50, 16009-16014 (2017)

11. Jafarzadeh, S., Jahed Motlagh, M.R., Barkhordari, M., Mirheidari, R.: A new Lyapunov based algorithm for tuning BELBIC controllers for a group of linear systems. In: 2008 16th Mediterranean Conference on Control and Automation. IEEE, June 2008

12. Garmsiri, N., Najafi, F.: Fuzzy tuning of brain emotional learning based intelligent controllers. In: 2010 8th World Congress on Intelligent Control and Automation. IEEE, July 2010

13. Jafari, M., Mohammad Shahri, A., Hamid Elyas, S.: Optimal tuning of brain emotional learning based intelligent controller using clonal selection algorithm. In: ICCKE 2013. IEEE, October 2013

14. Valizadeh, S., Jamali, M.-R., Lucas, C.: A particle-swarm-based approach for optimum design of BELBIC controller in AVR system. In: International Conference on Control, Automation and Systems, COEX, Seoul, Korea, pp. 2679-2684, October 2008 
15. Valipour, M.H., Maleki, K.N., Ghidary, S.S.: Optimization of emotional learning approach to control systems with unstable equilibrium. In: Lee, R. (ed.) Software Engineering, Artificial Intelligence, Networking and Parallel/Distributed Computing. SCI, vol. 569, pp. 45-56. Springer, Cham (2015). https://doi.org/10.1007/9783-319-10389-1_4

16. El-Saify, M.H., El-Garhy, A.M., El-Sheikh, G.A.: Brain emotional learning based intelligent decoupler for nonlinear multi-input multi-output distillation columns. Math. Probl. Eng. 1-13, 2017 (2017)

17. Mei, Y., Tan, G., Liu, Z.: An improved brain-inspired emotional learning algorithm for fast classification. Algorithms 10(2), 70 (2017)

18. César, M.B., Coelho, J.P., Gonalves, J.: Evolutionary-based bel controller applied to a magneto-rheological structural system. Actuators 7(2), 29 (2018)

19. Kennedy, J., Eberhart, R.C.: Particle swarm optimization. In: Proceedings of the 1995 IEEE International Conference on Neural Network, pp. 1942-1948 (1995)

20. Shi, Y., Eberhart, R.C.: Parameter selection in particle swarm optimization. In: Porto, V.W., Saravanan, N., Waagen, D., Eiben, A.E. (eds.) EP 1998. LNCS, vol. 1447, pp. 591-600. Springer, Heidelberg (1998). https://doi.org/10.1007/ $\mathrm{BFb0040810}$ 